\title{
NONSEPARABILITY OF CERTAIN FINITE FACTORS
}

\author{
JACOB FELDMAN ${ }^{1}$
}

Let $\mathcal{A}$ be a finite ring of operators with center $Z$; let $\Gamma$ be the maximal ideal space of $Z$, and $f$ the natural isomorphism from $Z$ onto the continuous complex functions on $\Gamma$. Let tr denote the centervalued trace on $A$. For each $\gamma$ in $\Gamma$, we define an inner product $(\cdot, \cdot)_{\gamma}$ on $\mathcal{A}$ by $(A, B)_{\gamma}=f\left(\operatorname{tr}\left(B^{*} A\right)\right)(\gamma)$. The members $A$ of $\mathcal{A}$ such that $(A, A)_{r}=0$ clearly form a two-sided ideal in $\mathcal{A}$, which we call $\mathcal{X}_{\gamma}$. Let $\mathcal{A}_{\gamma}$ be the quotient algebra $\mathcal{A} / \mathcal{X}_{\gamma}$, and $\phi_{\gamma}$ the natural map from $\mathcal{A}$ onto $\mathcal{A}_{\gamma}$. Since $\mathcal{X}_{\gamma}$ is closed under the * operation, a ${ }^{*}$ operation is induced on $\mathcal{A}_{\gamma}$ as well. It is shown in [6] that $\mathcal{X}_{\gamma}$ is a maximal two-sided ideal, and that $A_{\gamma}$ is a finite $A W^{*}$ factor with numerical trace, the trace being given by $\tau\left(\phi_{\gamma}(A)\right)=f(\operatorname{tr}(A))(\gamma)$. It is shown in [2] that any trace on a finite $A W^{*}$ factor is automatically countably additive; therefore the results of [1] show that $\mathcal{A}_{\gamma}$ is weakly closed in its canonical representation: that is, its representation as left multiplication operators on the Hilbert space $\mathfrak{F C}_{\gamma}$ gotten by completing $\mathcal{A}_{\gamma}$ in the inner product $\left(\phi_{\gamma}(A), \phi_{\gamma}(B)\right)=\tau\left(\phi_{\gamma}(B){ }^{*} \phi_{\gamma}(A)\right)$ $=(A, B)_{\gamma}$.

We shall show that the only time when $\mathfrak{H}_{\gamma}$ is a separable Hilbert space is when it is trivially so: that the more common state of affairs is for $\mathfrak{H}_{\gamma}$ to be nonseparable. From the nonseparability of $\mathfrak{H C}_{\gamma}$, furthermore, it follows that $A_{\gamma}$ can have no representation whatsoever as operators on a separable Hilbert space.

Lemma. Let $\Gamma_{1}, \Gamma_{2}, \cdots$ be nonempty disjoint closed and open sets in $\Gamma$. Let $E_{n}=f^{-1}\left(\chi_{\Gamma_{n}}\right)$, where $\chi_{\Gamma_{n}}$ is the characteristic function of $\Gamma_{n}$. Suppose $A\left(E_{n}\right)$ has disjoint orthogonal equivalent projections $P_{0}^{n}, \cdots, P_{n-1}^{n}$ with $P_{0}^{n}+\cdots+P_{n-1}^{n}=E_{n}$. Let $\gamma$ be in the closure of $\cup_{n-1}^{\infty} \Gamma_{n}$, but not in $\bigcup_{i-1}^{\infty} \Gamma_{n}$ itself. Then $\mathfrak{K}_{\gamma}$ is nonseparable.

Proof. We shall exhibit a collection $\left\{A_{\bar{\beta}}\right\}$ of members of $\mathcal{A}$, where $\bar{\beta}$ ranges over the set of all sequences $\bar{\beta}=\left(\beta_{0}, \beta_{1}, \cdots\right)$ of zeros and ones, such that $\left\{\phi_{\gamma}\left(A_{\bar{\beta}}\right)\right\}$ is an orthonormal set in $\mathfrak{F}_{\gamma}$.

Define $\alpha_{h, k}^{i}$, where $0 \leqq i \leqq k, 0 \leqq h \leqq 2^{k}-1$, as follows: $\alpha_{h, k}^{i}=(-1)^{\left[h, 2^{i}\right]}$, where $[r]$ denotes the largest integer $\leqq$ the real number $r$. For fixed $k$, and $i<j$, we have:

Received by the editors March 15, 1955.

1 The author holds a National Science Foundation postdoctoral fellowship. 


$$
\begin{aligned}
\sum_{h=0}^{2^{k}-1} \alpha_{h, k}^{i} \alpha_{h, k}^{j} & =\sum_{h=0}^{2 k-1}(-1)^{\left[h / 2^{i}\right]}(-1)^{\left[h / 2^{j}\right]} \\
& =\sum_{l=0}^{2^{k-i}-1} \sum_{h=l 2^{j}}^{(l+1) 2^{j}-1}(-1)^{[h / 2 i]}(-1)^{[h / 2 j]} \\
& =\sum_{l=0}^{2 k-j-1}(-1)^{l} \sum_{h=l 2^{j}}^{(l+1)^{j}-1}(-1)^{[h / 2 i]}
\end{aligned}
$$

But $(-1)^{\left(h / 2^{i}\right)}$ is positive and negative with equal frequency as $h$ ranges from $l 2^{i}$ to $(l+1) 2^{j}-1$, so that $\sum_{h=0}^{2^{k}-1} \alpha_{h, k}^{i} \alpha_{h, k}^{j}=0$.

Let $k(s)$ be the largest integer $k$ such that $2^{k} \leqq s$. We now define $A_{n}^{i}$ in $\mathcal{A}\left(E_{n}\right)$, for any positive integer $n$, and $2^{i} \leqq n$ :

$$
A_{n}^{i}=\left(n / 2^{k(n)}\right)^{1 / 2}\left(\sum_{h=0}^{2^{k(n)}-1} \alpha_{h, k(n)}^{i} P_{h}^{n}\right) .
$$

Then $\operatorname{tr}\left(A_{n}^{i *} A_{n}^{j}\right)=0$ if $i \neq j$, and $\operatorname{tr}\left(A_{n}^{i *} A_{n}^{i}\right)=E_{n}$. Furthermore, $\left\|A_{n}^{i}\right\|$ $=\left(n / 2^{k(n)}\right)^{1 / 2} \leqq(2)^{1 / 2}$. Thus, given any sequence $i=\left(i_{1}, i_{2}, \cdots\right)$ with $2^{i_{n}} \leqq n$, the partial sums $A_{1}^{i_{1}}+\cdots+A_{n}^{i_{n}}$ converge strongly to a member $A^{i}$ of $A$. If $i$ and $j$ are two such sequences, and $i_{n} \neq j_{n}$ for all $n>n_{0}$, then $\operatorname{tr}\left(A_{1}^{i_{1} *} A_{j}^{j_{1}}+\cdots+A_{n}^{i_{n} *} A_{n}^{j_{n}}\right)=\operatorname{tr}\left(A_{1}^{i_{1} *} A_{1}^{j_{1}}+\cdots+A_{n_{0}}^{i_{n_{0}} *} A_{n_{0}}^{i_{n_{0}}}\right)$ for all $n>n_{0}$, so that, by continuity of the $\operatorname{trace} \operatorname{tr}\left(A^{i *} A^{j}\right)$ $=\operatorname{tr}\left(A_{1}^{i_{1} *} A_{1}^{j_{1}}+\cdots+A_{n_{0}}^{i_{n_{0}} *} A_{n_{0}}^{j_{n_{0}}}\right)$, and therefore $f\left(\operatorname{tr}\left(A^{i *} A^{\bar{j}}\right)\right)$ vanishes outside $\Gamma_{1} \cup \ldots \cup \Gamma_{n_{0}}$, and in particular, vanishes at $\gamma$; consequently, $\phi_{\gamma}\left(A^{i}\right)$ is orthogonal to $\phi_{\gamma}\left(A^{\bar{j}}\right)$. Furthermore, $A^{i *} A^{i} \geqq A_{n}^{i_{n} *} A_{n}^{i_{n}}$ for all $n$, so that $f\left(\operatorname{tr}\left(A^{i_{*} *} A^{j}\right)\right)(\delta) \geqq f\left(\operatorname{tr}\left(A_{n}^{i_{n} *} A_{n}^{i_{n}}\right)\right)(\delta)=1$ for $\delta \in \Gamma_{n}$; and finally, $1 \geqq \operatorname{tr}\left(A_{1}^{i_{1} *} A_{1}^{i_{1}}+\cdots+A_{n}^{i_{n} *} A_{n}^{i_{n}}\right)$ for all $n$, so that $1 \geqq \operatorname{tr}\left(A^{i *} A^{i}\right)$. Thus $f\left(\operatorname{tr}\left(A^{i *} A^{i}\right)\right)(\delta)=1$ in $\bigcup_{n=1}^{\infty} \Gamma_{n}$, and therefore also in the closure of $\bigcup_{n=1}^{\infty} \Gamma_{n}$, and in particular for $\delta=\gamma$.

Now, if $n \geqq 4$ let $l(n)=k(k(n))-1$, and let $i_{n}(\bar{\beta})=2^{l(n)} \beta_{0}+2^{l(n)-1} \beta_{1}$ $+\cdots+2 \beta_{l(n)-1}+\beta_{l(n)}$, for any dyadic sequence $\bar{\beta}=\left(\beta_{0}, \beta_{1}, \cdots\right)$; if $n=1,2,3$ let $i_{n}(\bar{\beta})=1$. If $\bar{\beta} \neq \bar{\alpha}$, then $i_{n}(\bar{\beta})$ can equal $i_{n}(\bar{\alpha})$ only for finitely many $n$. Further, $\bar{i}_{n}(\bar{\beta}) \leqq 2 \cdot 2^{l(n)} \leqq k(n)$, so $2^{i_{n}(\bar{\beta})} \leqq n$. Then if $\bar{i}(\bar{\beta})=\left(i_{1}(\bar{\beta}), i_{2}(\bar{\beta}), \cdots\right)$, we write $A_{\bar{\beta}}$ for $A^{\bar{i}(\bar{\beta})}$. The set $\left\{\phi_{\gamma}\left(A_{\bar{\beta}}\right)\right\}$ is then our required orthonormal set in $\mathfrak{H C}_{\gamma}$.

If $\mathcal{B}$ is a finite factor with numerical trace $\tau$, and $\mathfrak{H C}$ is the Hilbert space gotten by completing $\mathbb{B}$ in its $\tau$-norm, then $\mathscr{B}$ has coupling constant $t^{2}$ equal to 1 in its canonical representation $B \rightarrow \lambda(\mathbb{B})$ on $\mathfrak{H C}$. Any 1-1 representation $\psi$ of $B$ as a ring of operators with coupling constant $\leqq 1$ can be constructed (up to unitary equivalence) by choosing an appropriate projection $P$ in $\lambda(\mathscr{B})^{\prime}$ and restricting $\psi(B)$ to

\footnotetext{
2 For definition and properties of the coupling constant, see [4].
} 
$P \mathcal{H}$; and if $\mathcal{F C}$ is nonseparable, then since $\lambda(B)$ must be type $\mathrm{II}_{1}$, and hence also $\lambda(B)^{\prime}$, it follows that $P \mathcal{F}$ is nonseparable. On the other hand, any representation of $B$ with coupling constant $\geqq 1$ can be constructed (again, up to unitary equivalence) by using a Hilbert space which has $\mathfrak{H C}$ as a subspace, and therefore is again nonseparable if $\mathfrak{H C}$ is. Thus, if $\mathcal{H}$ is nonseparable, $\mathbb{B}$ has no isomorphic representation as a ring of operators on a separable Hilbert space.

THEOREM. (a) If $\{\gamma\}$ is open, then $\mathfrak{H}_{\gamma}$ is separable if and only if $A(E)$ can be isomorphically represented as a ring of operators on a separable Hilbert space, where $E$ is $f^{-1}\left(\chi_{\{\gamma\}}\right)$. (b) If $\{\gamma\}$ is not open, then $\mathrm{F}_{\gamma}$ is separable if and only if there is a closed and open set $\Gamma_{0}$ containing $\gamma$ and such that $A\left(E_{0}\right)$ is n-homogeneous for some positive integer $n$, where $E_{0}$ is $f^{-1}\left(\chi_{\Gamma_{0}}\right)$.

Proof. (a) is evident from the discussion preceding this theorem and the fact that $\mathcal{H}_{\gamma}$ is precisely the Hilbert space of the canonical representation of the factor $A(E)$.

If $\{\gamma\}$ is not open, and $\Gamma_{0}$ exists as in the condition in (b), then, by using the structure theory of [3], it is not difficult to see that $A_{\gamma}$ is isomorphic to the algebra of $n \times n$ complex matrices, and therefore that $\mathcal{F C}_{\gamma}$ has finite dimension $n^{2}$.

Suppose, finally, that $\{\gamma\}$ is not open, but that no such $\Gamma_{0}$ exists. $\Gamma$ can be split in to disjoint closed and open sets $\Gamma_{I}$ and $\Gamma_{I I}$, corresponding to the split-up of the identity of $\mathcal{A}$ into central projections $E_{\mathrm{I}}$ and $E_{\mathrm{II}}$ of type I and type II respectively.

(i) Suppose $\gamma$ is in $\Gamma_{I}$. Then, by the structure theory of type I algebras, as described in [3], there are disjoint closed and open subsets $\Gamma_{1}, \Gamma_{2}, \cdots$ of $\Gamma_{I}$ such that, denoting by $E_{i}$ the projection $f^{-1}\left(\chi_{\Gamma_{i}}\right)$, we have $\sum_{n=1}^{\infty} E_{n}=I$, and $A\left(E_{n}\right)$ is $n$-homogeneous. By hypothesis, $\gamma$ is not in any of the $\Gamma_{n}$; but $\gamma$ is in the closure of $\bigcup_{n=1}^{\infty} \Gamma_{n}$, since this is all of $\Gamma_{\mathrm{I}}$. Furthermore, since $A\left(E_{n}\right)$ is $n$-homogeneous, it has $n$ orthogonal equivalent projections $P_{0}^{n}, \cdots, P_{n-1}^{n}$ whose sum is $E_{n}$. Therefore the conditions of the preceding lemma are satisfied, and $\mathfrak{H}_{\gamma}$ is nonseparable.

(ii) Suppose $\gamma \in \Gamma_{\mathrm{II}} . \Gamma_{\mathrm{II}}$ has some perfect measure $\mu$, as shown in [5]. Since $\gamma$ is not isolated, it is first category, and $\mu(\{\gamma\})=0$. Therefore there is a descending sequence $\Delta_{1}, \Delta_{2}, \ldots$ of closed and open sets containing $\gamma$, with $\mu\left(\Delta_{n}\right)<1 / 2^{n}$. Let $\Gamma_{1}=\Gamma_{I I}-\Delta_{1}$, and, inductively, $\Gamma_{n+1}=\Gamma_{\mathrm{II}}-\left(\Gamma_{1} \cup \ldots \cup \Gamma_{n} \cup \Delta_{n+1}\right)$. Then the $\Gamma_{n}$ are disjoint closed and open sets, and the complement of $\bigcup_{n-1}^{\infty} \Gamma_{n}$ is contained in $\bigcap_{n-1}^{\infty} \Delta_{n}$, hence has measure zero, so that $\bigcup_{n-1}^{\infty} \Gamma_{n}$ is dense in $\Gamma_{\text {II }}$. Furthermore, $\gamma$ is not in any of the $\Gamma_{n}$. And finally, if $E_{n}=f^{-1}\left(\chi_{\Gamma_{n}}\right)$, then $A\left(E_{n}\right)$ is 
finite and type II, so that $\mathcal{A}\left(E_{n}\right)$ has $n$ orthogonal, equivalent projections $P_{0}^{n}, \cdots, P_{n-1}^{n}$. Therefore again the hypotheses of our lemma are satisfied, and $\mathcal{H}_{\boldsymbol{\gamma}}$ is inseparable.

This completes the proof.

\section{REFERENCES}

1. J. Feldman, Embeddings of $A W^{*}$ algebras, to appear in Ann. of Math.

2. T. Iwamura, On continuous geometries, I, Jap. J. Math. vol. 9 (1944) pp. 57-71.

3. I. Kaplansky, Algebras of type I, Ann. of Math. vol. 56 (1952) pp. 460-472.

4. F. J. Murray and J. von Neumann, On rings of operators IV, Ann. of Math. vol. 44 (1943) pp. 716-808.

5. I. E. Segal, Decompositions of operator algebras I, Memoirs of the American Mathematical Society, no. 9, 1951.

6. F. B. Wright, $A$ reduction for algebras of finite type, Ann. of Math. vol. 60 (1944) pp. 560-570.

INSTITUte for Advanced Study 